\title{
Large Eddy Simulations for Indirect Combustion Noise Assessment in a Nozzle Guide Vane Passage
}

\author{
${\text { Alessandro } \text { Ceci }^{1} \text { (D) } \cdot \text { Romain Gojon }^{1,2} \cdot \text { Mihai Mihaescu }}^{1}$ \\ Received: 4 December 2017 / Accepted: 4 July 2018 / Published online: 21 August 2018 \\ (C) The Author(s) 2018
}

\begin{abstract}
The combustion noise in aero-engines is known to originate from two different sources. First, the unsteady heat release in the combustion chamber generates the direct combustion noise. Second, hot and cold spots of air generated by the combustion process are convected and accelerated by the turbine stages and give rise to the so-called indirect combustion noise. The present work targets, by using a numerical approach, the generation mechanism of indirect combustion noise for a simplified geometry of a turbine stator passage. Periodic temperature fluctuations are imposed at the inlet, permitting to simulate hot and cold packets of air coming from the unsteady combustion. Three-dimensional Large Eddy Simulation (LES) calculations are conducted for transonic operating conditions to evaluate the blade acoustic response to the forced temperature perturbations at the inlet plane. Transonic conditions are characterized by trailing edge expansion waves and shocks. It is notably shown that their movement can be excited if disturbances with a particular frequency are injected in the domain.
\end{abstract}

Keywords CFD · LES · Aeroacoustics · Indirect combustion noise

$\begin{array}{ll}\text { Nomenclature } \\ A & \text { amplitude of the entropy wave } \\ f_{1} & \text { forcing frequency of the entropy wave } \\ \Delta z & \text { cell width in z-direction } \\ L_{z} & \text { total mesh depth in z-direction } \\ C_{a x} & \text { blade axial chord } \\ H & \text { blade to blade distance } \\ p & \text { fluid static pressure }\end{array}$

Alessandro Ceci

aceci@kth.se

Mihai Mihaescu

miahi@mech.kth.se

1 KTH Royal Institute of Technology, 11428 Stockholm, Sweden

2 ISAE-SUPAERO, 31055 Toulouse, France 


\begin{tabular}{ll}
$p_{2}$ & face averaged outlet fluid static pressure \\
$p_{0}$ & fluid total pressure \\
$p_{01}$ & inlet fluid total pressure \\
$T$ & fluid static temperature \\
$T_{1}$ & inlet fluid static temperature \\
$T_{01}$ & inlet fluid total temperature \\
$y^{+}$ & non-dimensional near wall distance \\
$M_{i n}$ & inlet fluid Mach number \\
$M_{i s}$ & isentropic fluid Mach number \\
$\gamma$ & specific heats ratio \\
$c_{p}$ & specific heat at constant pressure \\
$x$ & axial coordinate \\
$y$ & azimuthal coordinate \\
$\Pi$ & fluid total pressure ratio \\
$t$ & time \\
$w^{s}$ & entropy wave \\
$w^{+}$ & transmitted acoustic wave \\
$w^{-}$ & reflected acoustic wave \\
$w_{1,2}^{ \pm, s}$ & acoustic/entropy wave at plane $1 / 2$ \\
$s$ & fluid entropy \\
$p^{\prime}$ & fluid static pressure fluctuation \\
$T^{\prime}$ & fluid static temperature fluctuation \\
$\bar{p}$ & time averaged fluid static pressure \\
\hline$T$ & time averaged fluid static temperature \\
$\tau$ & non-dimensional time \\
$U$ & velocity magnitude calculated from $M_{i s}=0.95$ \\
$D$ & trailing edge diameter \\
$R e f^{-}$ & acoustic reflection coefficient \\
$T r$ & acoustic transmission coefficient \\
$T r^{s}$ & entropy transmission coefficient \\
&
\end{tabular}

\section{Introduction}

Jet noise suppression technologies for aero-engines are currently available and implemented. Thus, the research attention was directed recently towards the relative importance of other noise sources in jet engines, e.g. indirect combustion (or entropy) noise. Its source was early investigated in the works of Candel [1] and Marble \& Candel [2]. They proposed an analytical model for the convection of non-uniform temperature regions through a nozzle in several configurations. In 1977, Cumpsty and Marble [3] proposed an analytical method, based on the actuator disk theory, for the evaluation of entropy noise through several turbine stages. Leyko et al. [4] analysed the wave generation and transmission mechanisms from a numerical perspective. In their work, they assessed the range of validity of the compact assumption for a stator blade row, comparing the analytical results with simulation data. In this paper, a transonic off-design condition of a nozzle guide vane (NGV) is analysed. The aim of the present work is to assess the indirect combustion noise generation mechanism using 3D LES calculations. A simplified topology will be adopted in the simulations in order to have a deeper physical comprehension of the phenomenon. The derived acoustic reflection and transmission coefficients will be compared with the ones predicted by the 
analytical model of Cumpsty and Marble [3]. Furthermore, singular flow features arising from transonic operating conditions are captured by the analysis of the frequency content of two-dimensional slices sampled during the simulation. The first part of the analysis will focus on the validation of the numerical solver against experimental results available for the baseline case without incoming disturbances. The second part, instead, will move towards the indirect combustion noise mechanism assessment.

\section{Geometry and Mesh}

The geometry adopted in the present study consists in a simplified topology of a real NGV cascade, for which experimental data were acquired by Yasa et al. [5] in a transonic test tunnel facility. The annular sector cascade was designed by Siemens Industrial Turbomachinery AB in 1998 and the guide vanes have identical geometry as the NGVs used in the first stage High Pressure Turbine (HPT) of a Siemens gas turbine. The complete 3D data of the studied NGV can be found in the work of Yasa et al. [5]. The simplification consists in adapting a linear cascade model as a representation of the 3D annular NGV cascade at mid-span. Afterwards, the mesh is built around the central unwrapped airfoil. It consists in a structured hexahedral-mesh with 18 blocks adapted manually to the high curvature stator blade. These hexa-cells are aligned along the local flow direction with the aim of capturing flow gradients in an accurate manner. The computational domain together with the mesh blocking strategy can be visualized in Fig. 1. For the LES calculations this $2 \mathrm{D}$ topology is extruded in the spanwise flow direction for capturing 3D effects. Periodic boundary conditions at the upper and lower boundaries are used in order to simulate an infinite linear cascade. Periodic boundary conditions are also applied at the extruded faces to model a channel of infinite width. Note that the height of the annulus enclosing the guide vanes is one order of magnitude lower than the inner radius of the cascade [6], thus a linear cascade model can be adopted. The 2D mesh is hence extruded with 75 layers of chosen width $\Delta z=0.0001[\mathrm{~m}]$. The width of the cell in the spanwise direction is based on the transverse Taylor micro-scale $\approx 0.0001[\mathrm{~m}]$. The total mesh depth in the spanwise direction $L_{z}$ is equal to $0.1095 C_{a x}$, where $C_{a x}$ represents the axial cord length, which is equal to 0.0685 [m]. The blade to blade distance $H$ is, instead, equal to $0.08345[\mathrm{~m}]$, which is also the distance between the upper and lower periodic boundaries. Sponge zones with gradual grid stretching are used at the inlet and at the outlet boundaries in order to damp
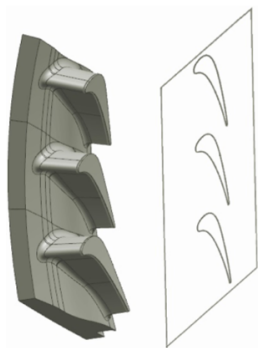

(a)

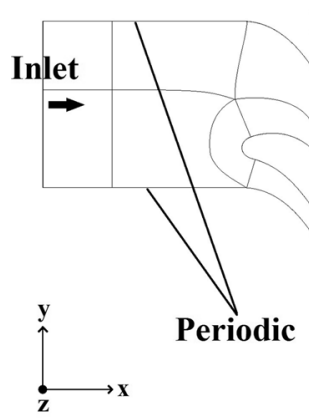

NGV Blade: Wall

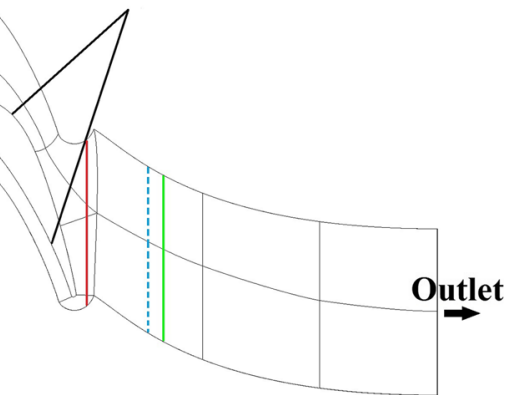

(b)

Fig. 1 Mesh topology a and blocking strategy $\mathbf{b}$ for the NGV linear cascade as a model of the 3D annular NGV cascade at mid-span 
spurious reflections. The total number of elements associated with the $3 \mathrm{D}$ domain is about 55.5 millions.

\section{Numerical Setup}

The numerical simulations are performed using the fully coupled density based solver dbnsTurbFoam, developed within the foam-extend framework ${ }^{1}$. It is an open-source, cellcentred, finite volume solver for compressible flows. The compressible Navier-Stokes equations are solved in their conservative form, which is necessary for capturing phenomena characteristic to transonic flows [7, 8]. The solver adopts the so-called method of lines to decouple spatial and temporal discretizations, reducing the governing equations to a system of ordinary differential equations (ODEs) [9]. The spatial convective discretization is performed with the Rusanov flux scheme [10], which is an approximation of the exact Rienman fluxes. This scheme is first order in space but the solver dbnsTurbFoam interpolates linearly the state from the cell center to the face center which makes the method of second order. Moreover, in order to avoid spurious oscillations near regions with sharp gradients, the Barth Jespersen limiter [11] is applied. The time integration is performed using an explicit 4-stage low-storage Runge-Kutta algorithm. The solver has been previously validated on two canonical compressible flow simulations by Chandramouli et al. [12]. The LES simulations are initialized with the solution of the 2D Reynolds Averaged Navier-Stokes (RANS) equations using Favré averaging. The $k-\omega S S T$ compressible turbulence model was adopted for the 2D RANS calculations. The LES turbulence model relies, instead, on the one-equation model for the subgrid-scale kinetic energy [13].

\section{Baseline Case}

The baseline case of the present study refers to the numerical simulation matching the off-design transonic condition of the experiment [5], without any inflow disturbances. The boundary conditions at the inlet are specified in terms of total pressure $p_{01}=187100[\mathrm{~Pa}]$ and total temperature $T_{01}=304.51[\mathrm{~K}]$. The inflow velocity direction is perpendicular to the inlet boundary patch.

At the outlet, a face averaged static pressure of $p_{2}=104370[\mathrm{~Pa}]$ is imposed. The airfoil walls are treated as no-slip adiabatic walls. Due to the fine refinement region near the wall $\left(y^{+} \approx 5\right)$, the use of wall function has been avoided. A snapshot of the instantaneous Mach, pressure and temperature fields (LES data) can be visualized in Fig. 2. The Mach number field shows the essential flow features of the simulated case: the flow is subsonic at the inlet of the domain with the local Mach number equal to $M_{i n}=0.15$, matching the experimental conditions.

Then, the flow is accelerated up to sonic condition at the throat of the nozzle guide vane passage. Passed the throat the flow interacts with compression and expansion waves emitted from the shed vortices at the trailing edge (seen from Fig. 2b). These waves are also impinging on the suction side and then are reflected back towards the normal direction of the blade surface. The flow accelerates further on the suction side just above sonic conditions according to a Prandtl-Mayer expansion. Towards the end of suction side, one can observe

\footnotetext{
${ }^{1}$ https://sourceforge.net/p/openfoam-extend/wiki/Home/
} 


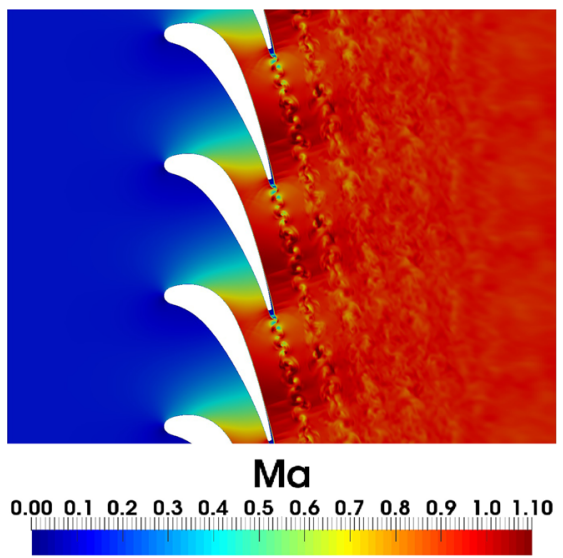

(a)

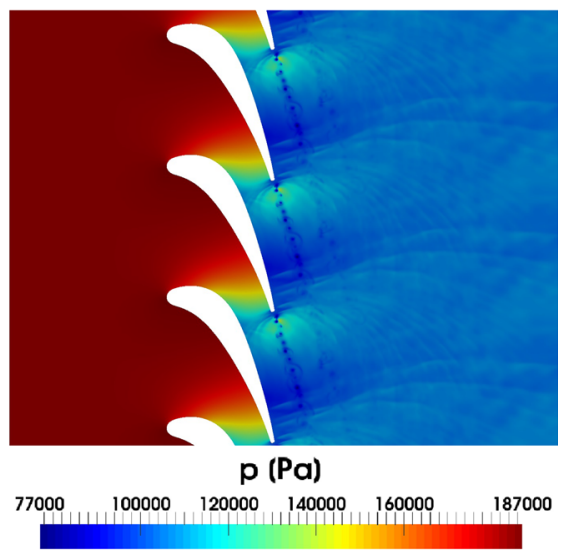

(b)

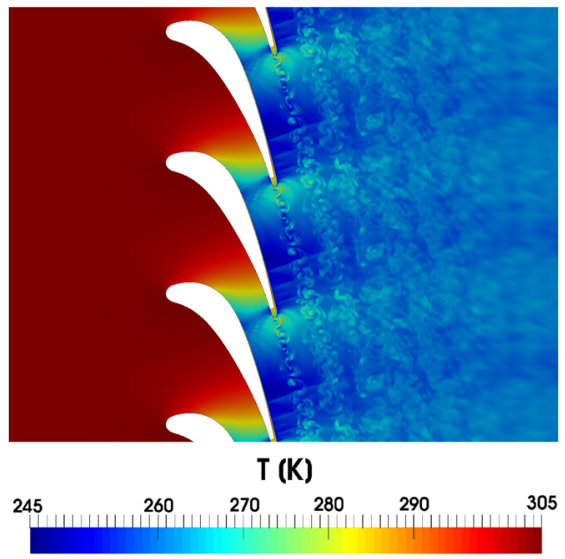

(c)

Fig. 2 Instantaneous Mach number $\mathbf{a}$, pressure $\mathbf{b}$ and temperature $\mathbf{c}$ fields at midspan, LES data

a system of weak shocks present, unsteady and moving with its own dynamics. The results from the 2D RANS (used for the initialization) and the 3D LES are then compared with the experimental data. In Fig. $3 \mathrm{a}$ and $\mathrm{b}$, the NGV blade loading and the circumferential pressure distribution at $x / C_{a x}=40 \%$ downstream from the blade are presented in terms of the isentropic Mach number $M_{i s}$, which is defined as

$$
M_{i s}=\sqrt{\frac{2}{\gamma-1}\left[\left(\frac{p_{01}}{p}\right)^{\frac{\gamma-1}{\gamma}}-1\right]} \text {; }
$$

where $p_{01}$ is the inlet total pressure, $p$ is the local static pressure and $\gamma$ is the ratio of the specific heats. The blade loading is expressed as a function of the axial normalized coordinate $x / C_{a x}$ while the circumferential pressure distribution is expressed as a function of the vane phase, as presented in the work by Yasa et al. [5]. For the pressure side, notably a very good agreement is found between the experiments and both the numerical simulations. The matching is also fairly good on the suction side, with a slight underprediction after 
Fig. 3 NGV blade loading $\mathbf{a}$ and downstream pressure distribution at $x / C_{a x}=40 \%$, line (_ - ) in Fig. 1b b; (•) experimental data; (_ - ) 2D RANS; (_) 3D LES

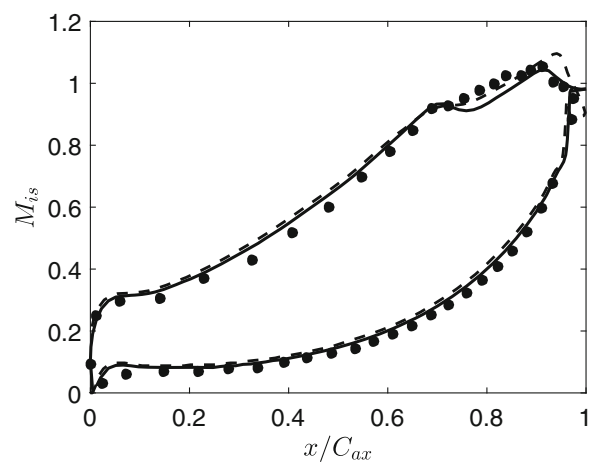

(a)

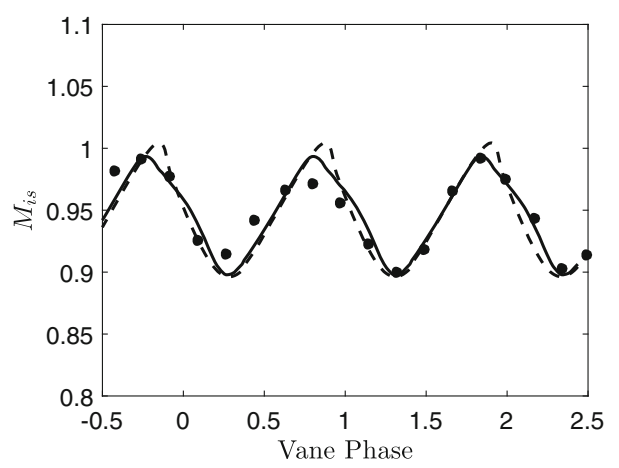

(b)

the throat region. The pressure distribution along the blade involves different features due to the physics of the flow field. On the pressure side the flow is accelerated up to sonic conditions at almost the end of the blade; the entire pressure sides behaves like a converging portion of a nozzle. On the suction side, the flow accelerates up to chocking conditions until $x / C_{a x}=0.75$. Then, the Mach number is increased due to a supersonic expansion on the final part of the blade. Finally, the Mach number drops just below sonic conditions due to the presence of the weak normal shock. The location of the shock is just slightly overpredicted by the 2D RANS. Regarding the pressure distribution downstream of cascade, Fig. 3b, the topology of the domain allows, by construction, to reproduce only one passage, and the subsequent resulting trend is periodic. The experimental data are available for almost three complete passages. It can be noticed that the LES data are in perfect agreement in phase 2; 2D RANS also show an overall good agreement in that region. The mismatch for few other locations is due to the fact that experimental data are not perfectly periodic. Then, the total pressure ratio is defined as

$$
\Pi=\frac{p_{0}}{p_{01}},
$$

where $p_{0}$ is the local total pressure. This ratio is plotted in Fig. 4 for two locations downstream of the passage, at $x=7.1 \% C_{a x}$ and at $x=48.4 \% C_{a x}$ respectively. The same circumferential coordinate $y /$ Pitch as Yasa et al. [5] is adopted. The increasing wake region extension downstream the NGV passage can be clearly seen from the pictures. The total pressure losses are also reduced thanks to the enhanced mixing in the wake region. The 
Fig. 4 Total pressure ratio: at $x / C_{a x}=0.071$, line (_) in Fig. $1 \mathrm{~b}$ a and at $x / C_{a x}=0.484$, line (_) in Fig. 1b b; (•) experimental data; (_ - ) 2D RANS; (—) 3D LES

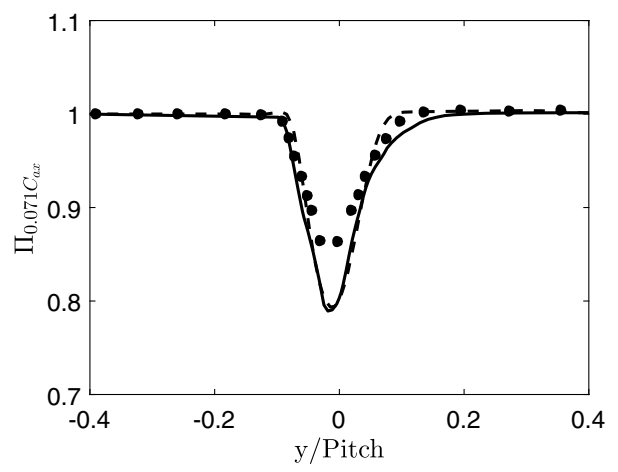

(a)



(b)

asymmetric profile at the closest location to the trailing edge is very well captured by the 3D LES. The resulting profile from the 2D RANS appears slightly more symmetric. In the furthest location, the 3D LES exhibits a much closer profile to the experimental data than the 2D RANS, for which the wake width is underpredicted. Overall, a fair agreement is found for both 3D LES and 2D RANS results in terms of wake width at $7.1 \% C_{a x}$ downstream the passage. Instead, At $48.4 \% C_{a x}$ the 3D LES results agree better with experimental data than the RANS. Finally, regarding the maximum pressure losses, both simulation results overpredict them at $7.1 \% C_{a x}$ while the $3 \mathrm{D}$ LES results are closer to the experimental ones at $48.4 \% C_{a x}$.

\section{Forced Case}

Indirect combustion noise results from non-isentropic temperature fluctuations that are accelerated at the combustor outlet [14].

In the present LES, temperature disturbances are periodically forced at inlet as planar waves for simulating entropy waves, according to:

$$
\tilde{T}_{1}=T_{1}+T_{1} A \sin \left(2 \pi f_{1} t\right)
$$

where $\tilde{T}_{1}$ is the oscillating static temperature, $T_{1}$, the inlet static temperature of the baseline case, $A$ the amplitude of the disturbance and $f_{1}$ the temporal frequency. The amplitude 
$A$ has been set equal to $4.8 \%$ and the the frequency $f_{1}$ to $1000[\mathrm{~Hz}]$. The choice of the frequency is dictated by the high computational cost of the 3D LES. However, this frequency is still in the limit range of real case scenarios; previous studies in literature indicated that combustion noise has its peak intensity typically in the low frequency range around $200-$ $1000[\mathrm{~Hz}][15-18]$. The compact assumption states that the wavelength of the incoming entropy waves is much larger (by a factor of 10) than the blade axial chord. Still, similar simulations have been conducted by Papadogiannis et al. [19], which found that the limiting frequency for the validity of the compact assumption [3] is around 2200 [Hz]; which results in a less strict condition for a NGV blade in real case scenarios.

Snapshots of the instantaneous entropy wave field in a spanwise section can be visualized in Fig. 5 for both the baseline and the forced case. The entropy wave, denoted by the superscript ${ }^{s}$, is defined as $w^{s}=s^{\prime} / c_{p}=T^{\prime} / \bar{T}-(\gamma-1) p^{\prime} / \gamma \bar{p}$, where the primed quantities are respectively the entropy $\left(s^{\prime}\right)$, temperature $\left(T^{\prime}\right)$ and pressure $\left(p^{\prime}\right)$ fluctuation fields; the overlined ones are the time averaged temperature $(\bar{T})$ and pressure $(\bar{p})$ field, and $c_{p}$ and $\gamma$ are respectively the specific heat at constant pressure and the specific heats ratio. For the baseline case in Fig. 5a, the flow accelerates up to sonic conditions at the throat. Then the "cross"-like pattern of waves, shed from the trailing edge vortices, is present just after the throat. Finally a system of weak shocks is visible at the end of the blade. Figure 5b shows, instead, the features of the flow-field with planar entropy waves injected at the inlet. The planar forcing is revealed at the inlet by the presence of vertical stripes in the entropy fluctuation field. Once the waves approach the blades they get distorted by the large gradients of mean flow quantities across the vane, becoming aligned with the flow direction at the end of the passage. Then, one axial chord downstream from the blade, it is possible to see almostplanar entropy waves propagating downstream. A temporal fast Fourier transform (FFT) has been conducted for a set of two-dimensional snapshots of velocity, temperature and pressure fields, sampled at a frequency equal to four times the vortex shedding frequency, in order to avoid aliasing. This frequency is widely sufficient to capture possible features of the flow-field appearing at $f_{1}=1000[\mathrm{~Hz}]$. For the baseline and the forced case, the amplitude of the acoustic wave field $w^{ \pm}=p^{\prime} / \gamma \bar{p}$ is pictured in Fig. 6 from the FFT at the inlet forcing frequency $f_{1}$. The forced case, Fig. $6 \mathrm{~b}$, shows several regions of interest with noticeable amplitudes highlighted by the respective markers. No relevant amplitudes were

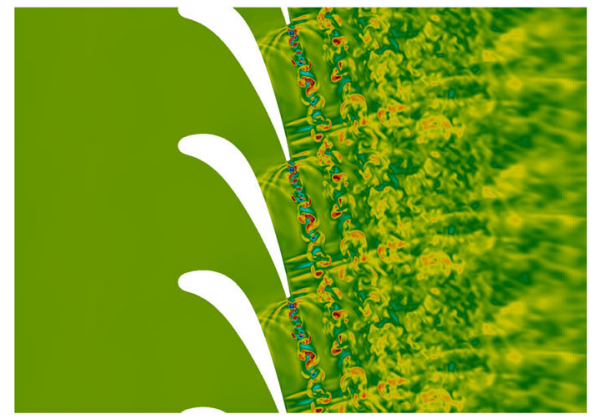

(a)

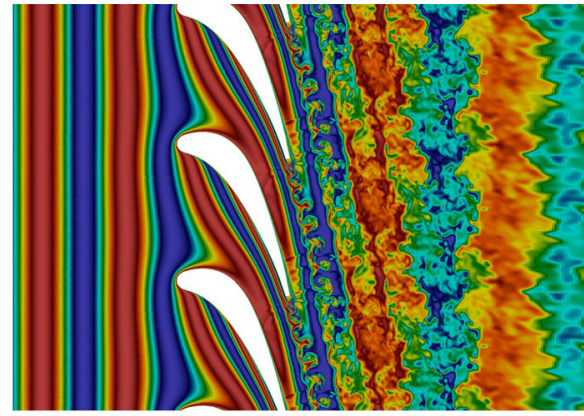

(b)



Fig. 5 Instantaneous entropy wave field for the baseline $\mathbf{a}$ and for the forced $\mathbf{b}$ case 

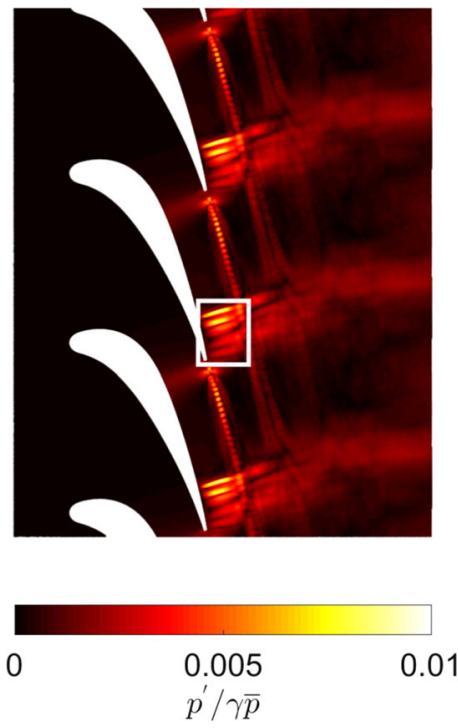

(a)
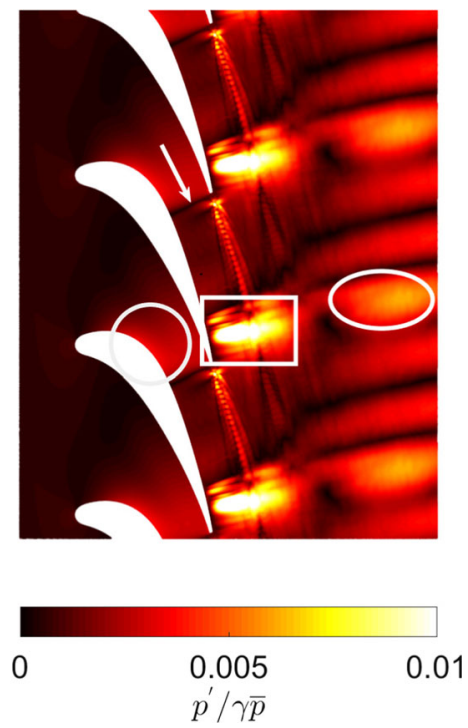

(b)

Fig. 6 Amplitude of the acoustic wave at $f_{1}=1000[\mathrm{~Hz}]$ for the unforced baseline case (a) and for the forced case (b)

instead detected for the baseline case of Fig. 6a at $f_{1}=1000[\mathrm{~Hz}]$, with the exception of a small region on the suction side of the blade, characterized by the presence of compression and expansion waves at the particular frequency $f_{1}$. For the forced case, a glowing yellowwhite area can be seen on the suction side, towards the end of the blade (white rectangle in Fig. 6b). This area is overlapped with the system of weak shocks, visible in the instantaneous Mach, pressure and temperature instantaneous flow-field of Fig. 2. This area is also visible in the baseline case of Fig. 6a, but with a less extended area of high pressure amplitudes. The convergent section of the vane passage also exhibits new notable amplitudes up to the throat section with respect to the baseline case (white circle of Fig. 6b). At the outlet (white ellipse of Fig. 6b), the acceleration of the entropy spots through the NGV passage and the acoustic waves generated on the suction side and propagated downstream, are strong enough to produce a significant pressure fluctuation trace with non-zero modulus [19]. A possible explanation can be that entropy waves, interacting with strong mean pressure and mean velociy gradients, give rise to an entropy-acoustic modal interaction. Considering for instance a weakly non linear Kovasznay decomposition [20,21] of all compressible flow variables, acoustic production due to acoustic-entropy coupling can arise due to scattering: i.e. the acoustic waves are scattered by the convected entropy spots passing through the nozzle. According to Kovasznay decomposition [20,21] the acoustic production scattering term is $\partial_{t}\left(\nabla \cdot s_{e} \mathbf{u}_{\mathbf{p}}\right)$, where $s_{e}$ is entropy perturbation of the entropy mode and $\mathbf{u}_{\mathbf{p}}$ is the velocity perturbation of the acoustic mode. Although no acoustic forcing is imposed by the entropy waves injected at the inlet, distinctive higher amplitudes of the acoustic waves in the whole domain reveal that a pressure mode with a distinctive peak at the frequency $f_{1}$ appears, confirming the indirect noise generation mechanism [19]. In the forced case, new regions of high amplitudes pressure fluctuations appear, which are not present in the baseline case. A 
dark line (white arrow in Fig. 6b) in the neighbourhood of the sonic throat identifies also a region of lower amplitude. In the convergent region, only the reflected waves are present because the waves created downstream of the sonic line cannot travel upstream. The attention will move now to the glowing area with the highest amplitude, in Fig. 6b. Entropy waves seem to strongly interact with the system of weak normal shocks, therefore a sampling line has been traced in this particular zone as sketched in Fig. 7. For this line, the pressure fluctuation field has been sampled in time for a number of 100 spatial points with the resolved frequency range of $50[\mathrm{KHz}]$. Then, the sampled field is projected on a space-time grid where the spatial coordinate is represented by the non-dimensional distance $x / C_{a x}$ and the temporal coordinate by a non dimensional time $\tau^{*}=t U / D, U$ being the velocity calculated from the Mach number $M_{i s}=0.95$ and $D$ the trailing edge diameter, equal to $0.0022[\mathrm{~m}]$. With this method the characteristic dynamics of the system of normal shocks can be identified and the present analysis will be conducted for both the baseline and the forced case. The pressure fluctuation field is expressed in terms of its non dimensional counterpart defined as the acoustic wave $w^{ \pm}$. Figure 8 shows the non dimensional acoustic wave projected on the space-time diagram for both the baseline and the forced case. Three regions of interest can be identified in the graph. Region 1 corresponds to the zone where the position of the shock changes slowly during time; this region has fairly similar characteristics for both the baseline and the forced case. The frequency content in Region 1 is in the range of $200-900[\mathrm{~Hz}]$. Region 2, instead, corresponds to the glowing zone for which the amplitude of the pressure fluctuations is the highest in Fig. 6. For the forced case in Fig. 8b, yellow-white stripes at regular intervals of time are visible in Region 2. The frequency of these intervals is evaluated by the means of a FFT at a fixed position in space: it corresponds exactly to the inlet forcing frequency $f_{1}=1000[\mathrm{~Hz}]$. This feature does not appear for the baseline case in Fig. 8a and the shock movement is not organized at any particular frequency of interest; its frequency content is instead in the range of $500-3000[\mathrm{~Hz}]$. Finally, Region 3 corresponds to the initial part of the wake, where compression and expansion waves are emitted by vortical structures and appear with higher frequencies. The structure of Region 3 is similar for

Fig. 7 Line for the analysis of shock dynamics

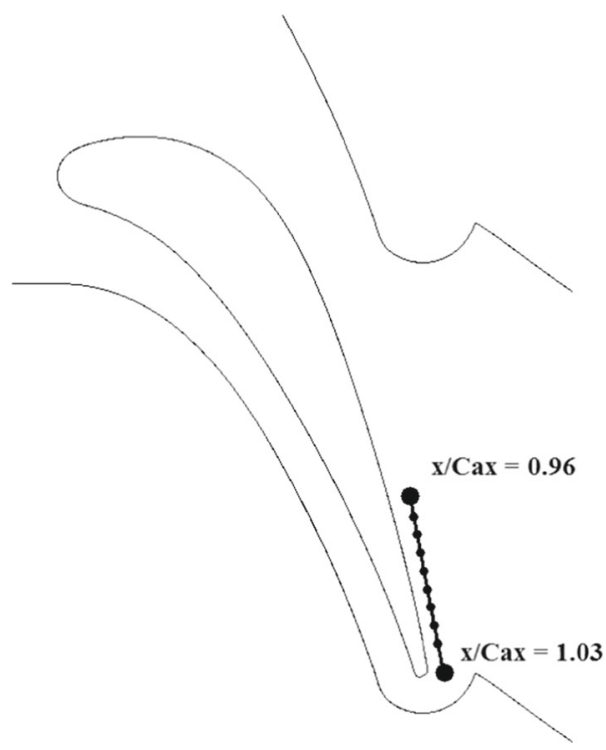






(a)

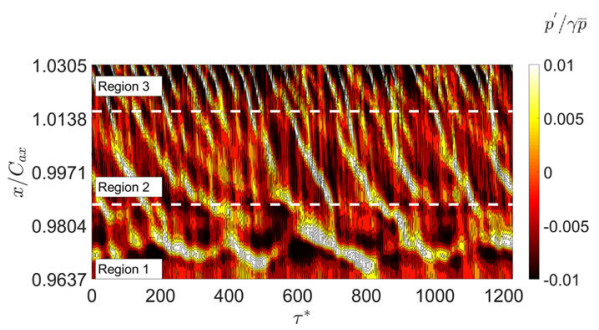

(b)

Fig. 8 Isocontours of $p^{\prime} / \gamma \bar{p}$ in the space-time diagram; baseline case $\mathbf{a}$; forced case $\mathbf{b}$

both cases and its dynamics is in the range of $3000-7000[\mathrm{~Hz}]$. These aspects of the shock dynamics in Region 2 justify the high amplitude found by the FFT at $1000[\mathrm{~Hz}]$. The shock movement mechanism, with shocks appearing and then vanishing, was documented as splitting mechanism, see the work of Panda [22] and the work of Risborg \& Soria [23]. Finally, the reflection coefficient $R e f^{-}=w_{1}^{-} / w_{1}^{s}$ and the trasmission coefficients $\operatorname{Tr}^{+}=w_{2}^{+} / w_{1}^{s}$, $\operatorname{Tr}^{s}=w_{2}^{s} / w_{1}^{s}$ are compared with the analytical results available in literature $[3,4,19]$.

The coefficients of interest are then evaluated on two planes parallel to the inlet boundary, respectively one at $x / C_{a x}=-0.65$ upstream (plane 1) and one at $x / C_{a x}=2.40$ downstream (plane 2) of the leading edge of the airfoil. The planes of interest can be visualized in Fig. 9. The acoustic reflection coefficient $\operatorname{Ref}^{-}$, denoted by the superscript ${ }^{-}$, is defined as the ratio between the reflected acoustic wave $w_{1}^{-}$and the incoming entropy wave $w_{1}^{s}$, both at plane 1 . On the other hand, the acoustic transmission coefficient $\operatorname{Tr}^{+}$, denoted by the superscript ${ }^{+}$, is defined as the ratio between the transmitted acoustic wave $w_{2}^{+}$at plane 2 and the incoming entropy wave $w_{1}^{s}$ at plane 1. Finally, the entropy transmission coefficient $T r^{s}$, denoted by the superscript ${ }^{s}$, is defined as the ratio between the transmitted entropy wave $w_{2}^{s}$ at plane 2 and the incoming entropy wave $w_{1}^{s}$ at plane 1 . The definition of the entropy and acoustic waves follows the one showed in Section 5. Table 1 summarizes the results for these coefficients.

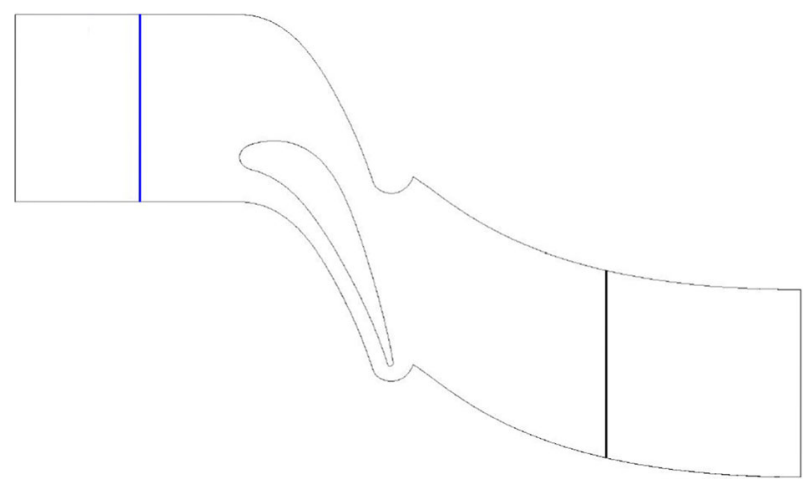

Fig. 9 Azimuthal planes adopted for the evaluation of reflection and transmission coefficients; (_) upstream plane $1,($ ) downstream plane 2 
Table 1 Reflection and transmission coefficients. Comparison between numerical simulations and analytical model

\begin{tabular}{lll}
\hline & analytical & CFD \\
\hline$\left|R e f^{-}\right|$ & 0.073 & 0.011 \\
$\left|\operatorname{Tr}^{+}\right|$ & 0.088 & 0.068 \\
$\left|\operatorname{Tr}^{s}\right|$ & 1 & 0.45 \\
\hline
\end{tabular}

\section{Conclusions}

The indirect combustion noise generation mechanism has been addressed by the means of 3D LES for a simplified nozzle guide vane passage of a high pressure turbine. The solver adopted for the simulations was first validated against experimental data available for offdesign transonic conditions without temperature forcing. Overall the numerical results are in very good agreement with experimental data. The larger discrepancies were only found in terms of maximum total pressure losses which might be due to the simplified 2D-extruded geometry adopted, for which the wake mixing might not be enhanced as in the real case scenario. In the second part of the work, a pulsating entropy wave-train was imposed at the inlet of the domain to simulate temperature non-uniformities coming from the combustion chamber. The resulting flowfield was analysed by the FFT of two-dimensional sections sampled during the simulation. At the forcing frequency imposed at the inlet, certain zones in the fluid respond with high amplitudes of the pressure fluctuations, which are not present in the baseline case. An interesting feature was observed regarding the system of shocks emitted from the trailing edge: their dynamics in a particular region (Region 2) was completely characterized by a frequency content at $1000[\mathrm{~Hz}]$, which is the inlet forcing frequency of the entropy waves. Finally, reflection and transmission coefficients were compared to analytical models available in literature [3]. While the theoretical approach overpredicts the noise generated, the 3D simulations show that the entropy waves are highly distorted by the passage and lose their strength. This can be addressed from the entropy wave transmission coefficient which is reduced by $55 \%$. The reflected acoustic wave, instead, is far weaker than the one predicted by the compact theory. Finally, the reflected acoustic wave is slightly lower than the analytical predicted one, but its amplitude is still strong. It is therefore the main contributor to the generated indirect combustion noise.

Acknowledgements The simulations were performed at the PDC Center for High Performance Computing (PDC-HPC) and at the High Performance Computing Center North (HPC2N), on resources provided by the Swedish National Infrastructure of Computing (SNIC).

\section{Compliance with Ethical Standards}

Conflict of interests The authors declare that they have no conflict of interest.

Open Access This article is distributed under the terms of the Creative Commons Attribution 4.0 International License (http://creativecommons.org/licenses/by/4.0/), which permits unrestricted use, distribution, and reproduction in any medium, provided you give appropriate credit to the original author(s) and the source, provide a link to the Creative Commons license, and indicate if changes were made.

Publisher's Note Springer Nature remains neutral with regard to jurisdictional claims in published maps and institutional affiliations. 


\section{References}

1. Candel, S.: Analytical Studies of Some Acoustic Problems of Jet Engines. PhD thesis (1972)

2. Marble, F.E., Candel, S.: Acoustic disturbances from gas non uniformities convected through a nozzle. J. Sound Vib. 55(2), 225-243 (1977)

3. Cumpsty, N.A., Marble, F.E.: The interaction of entropy fluctuations with turbine blade rows; a mechanism of turbojet engine noise. Proc. R. Soc. 357(1690), 323-344 (1977)

4. Leyko, M., Duran, I., Moreau, S., Nicoud, F., Poinsot, T.: Simulation and modeling of the waves transmission and generation in a stator blade row in a combustion-noise framework. J. Sound Vib. 333, 6090-6106 (2014)

5. Yasa, T., Fridh, J., Paniagua, G., Vogt, D.: Performance of a nozzle guide vane in subsonic and transonic regimes tested in an annular sector. ASME Turbo Expo 2010, GT2010-22901 (2010)

6. Atassi, H., Ali, A., Atassi, O.: Interaction of acoustic and vortical disturbances with an annular cascade in a swirling flow. Unsteady Aerodynamics, Aeroacoustics and Aeroelasticity of Turbomachines, pp. 247-259 (2006)

7. LeVeque, R.J.: Finite-Volume Methods for Hyperbolic Problems. Cambridge University Press (2002)

8. Lax, P., Wendroff, B.: Systems of conservation laws. Commun. Pure Appl. Math. 13(2), 217-237 (1960)

9. Blazek, J.: Computational Fluid Dynamics: Principles and Applications. No. v. 1. Elsevier (2001)

10. Rusanov, V.: "The calculation of the interaction of non-stationary shock waves and obstacles," USSR. Comput. Math. Math. Phys. 1(2), 304-320 (1962)

11. Barth, T., Jespersen, D.: The design and application of upwind schemes on unstructured meshes. In: 27 th Aerospace Sciences Meeting, p. 366 (1989)

12. Chandramouli, S., Gojon, R., Fridh, J., Mihaescu, M.: Numerical characterization of entropy noise with a density based solver. ETC2017-143 (2017)

13. Yoshizawa, A.: Bridging between eddy-viscosity-type and second-order turbulence models through a two-scale turbulence theory. Phys. Rev. E 48, 273-281 (1993)

14. Polifke, W., Paschereit, C., Döbbeling, K.: Constructive and destructive interference of acoustic and entropy waves in a premixed combustor with a choked exit. Int. J. Acoust. Vib. 6, 135-146 (2001)

15. Dowling, A.P., Mahmoudi, Y.: Combustion noise. Proc. Combust. Inst. 35(1), 65-100 (2015)

16. Hassan, H.: Scaling of combustion-generated noise. J. Fluid Mech. 66(3), 445-453 (1974)

17. Kotake, S., Takamoto, K.: Combustion noise: effects of the shape and size of burner nozzle. J. Sound Vib. 112(2), 345-354 (1987)

18. Ihme, M., Pitsch, H.: On the generation of direct combustion noise in turbulent non-premixed flames. Int. J. Aeroacoust. 11(1), 25-78 (2012)

19. Papadogiannis, D., Wang, G., Moreau, S., Duchaine, F., Gicquel, L., Nicoud, F.: Assessment of the indirect combustion noise generated in a transonic high-pressure turbine stage. J. Eng. Gas Turbine Power 138(4), 041503 (2016)

20. Chu, B.-T., Kovásznay, L.S.: Non-linear interactions in a viscous heat-conducting compressible gas. J. Fluid Mech. 3(05), 494-514 (1958)

21. Garnier, E., Adams, N., Sagaut, P.: Large Eddy Simulation for Compressible Flows. Springer Science \& Business Media (2009)

22. Panda, J.: Shock oscillation in underexpanded screeching jets. J. Fluid Mech. 363, 173-198 (1998)

23. Risborg, A., Soria, J.: High-speed optical measurements of an underexpanded supersonic jet impinging on an inclined plate. In: 28th International Congress on High-Speed Imaging and Photonics, pp. 71261F71261F. International Society for Optics and Photonics (2008) 